\title{
DEDUÇÃO DA EQUAÇÃO DA VARIANÇA ESPACIAL LATERAL PARA UMA NOVA FORMULAÇÃO DA FUNÇÃO DE AUTOCORRELAÇÃO LAGRANGIANA
}

Charles Rogério Rogério Paveglio Szinvelski ${ }^{2}$, Gervásio Annes Degrazia ${ }^{1}$, Lidiane Buligon²,

\author{
Lílian Piecha Moor ${ }^{1}$ \\ ${ }^{2}$ charless@ufsm.br \\ ${ }^{1}$ Departamento de Física, Universidade Federal de Santa Maria, RS-Brasil. \\ ${ }^{2}$ Departamento de Matemática, Universidade Federal de Santa Maria, RS-Brasil.
}

\section{RESUMO}

Neste trabalho deriva-se uma nova forma para o parâmetro de variância espacial lateral, $\sigma_{y}$ válida para diferentes tipos de turbulência na CLP.

\section{ABSTRACT}

IIn this paper derives a new form to the lateral spatial variance parameter, $\sigma_{y}$ valid for different types of turbulence in the PLC.

\section{INTRODUÇÃO}

A função de autocorrelação lagrangiana é uma quantidade estatística fundamental na descrição de escoamentos turbulentos (Taylor, 1921). Esta função é uma medida estatística das estruturas coerentes encontradas por um conjunto de partículas de fluido à medida que elas se dispersam no campo turbulento (Armenio et al, 1999). Normalmente, a maioria dos estudos teóricos e práticos em turbulência descreve relações fundamentais e funções de autocorrelação lagrangianas para um campo turbulento bem desenvolvido (Hinze, 1975; Pasquill e Smith, $1983 ; \ldots)$.

Em seu trabalho clássico sobre a teoria de difusão estatística da turbulência, Taylor em 1921, considerou uma forma exponencial para a função de autocorrelação lagrangiana dada pela seguinte expressão:

$$
\rho_{L_{v}}=\exp (\quad)
$$

onde, $\tau$ é o tempo de correlação e $T_{L_{v}}$ é a escala de tempo integral lagrangiana associada as estruturas coerentes caracterizando uma turbulência bem desenvolvida. 
A substituição de Eq. (1) no modelo de difusão estatístico de Taylor resulta na seguinte expressão para o variância espacial lateral $\sigma^{2}{ }_{y}$,

$$
\sigma_{y}^{2}=2 \sigma_{v}^{2} T_{L_{v}}\left[\frac{1}{T_{L_{v}}}-1+\exp \left(\frac{-t}{T_{L_{v}}}\right)\right]
$$

onde $\sigma_{v}$ é o desvio padrão da velocidade lateral turbulenta A partir da Eq. (2), relações funcionais são deduzidas. Como exemplo, a taxa de dissipação turbulenta $\varepsilon$, utilizada em parametrizações turbulentas aplicadas em modelos de dispersão lagrangianos.

A função de autocorrelação lagrangiana exponencial (Eq. (1)), aplica-se apenas aos casos de turbulência bem desenvolvida. Porém, em uma situação de velocidade de vento baixa, com baixa frequência nas oscilações do vento horizontal (o que caracteriza o fenômeno de meandro), a turbulência é dita fraca, descaracterizando a premissa de sua aplicabilidade.

Nesta sentido, pretende-se construir uma formulação para a função de autocorrelação lagrangiana que contemple, mediante a inserção de parâmetros adequados, as possíveis situações de ocorrências de turbulência na CLP. O ponto de partida, para este fim, utiliza-se da sugestão de Phillips e Panofsky (1982) (ou Pasquill e Smith (1983)), para a função de autocorrelação em uma situação de turbulência bem desenvolvida, dada por $\rho_{L_{v}}(\tau)=$ $(1+p \tau)^{-2}$, com $p=\frac{1}{\left(1+m^{2}\right) T_{L_{v}}}$.Entretanto, para atender as variadas manifestações de turbulência na CLP, introduziu-se a expressãocos $(q \tau)$,param $\geq 0$ (quantidade adimensional que controla a frequência de oscilação do vento horizontal, meandro $[q=m p]$ ), resultando em

$$
\rho_{L_{v}}(\tau)=\frac{\cos (q \tau)}{(1+p \tau)^{2}}
$$

Salienta-se que essa nova formulação atenderá a descrição de lóbulos negativos de uma função de autocorrelação que descreva o fenômeno de meandro; e seu comportamento assemelha-se a função de autocorrelação lagrangiana sugerida por Frenkiel (1953),

$$
\rho_{L_{v}}(\tau)=\exp \left(-\tau / T_{L_{v}}\right) \cos (q \tau)
$$



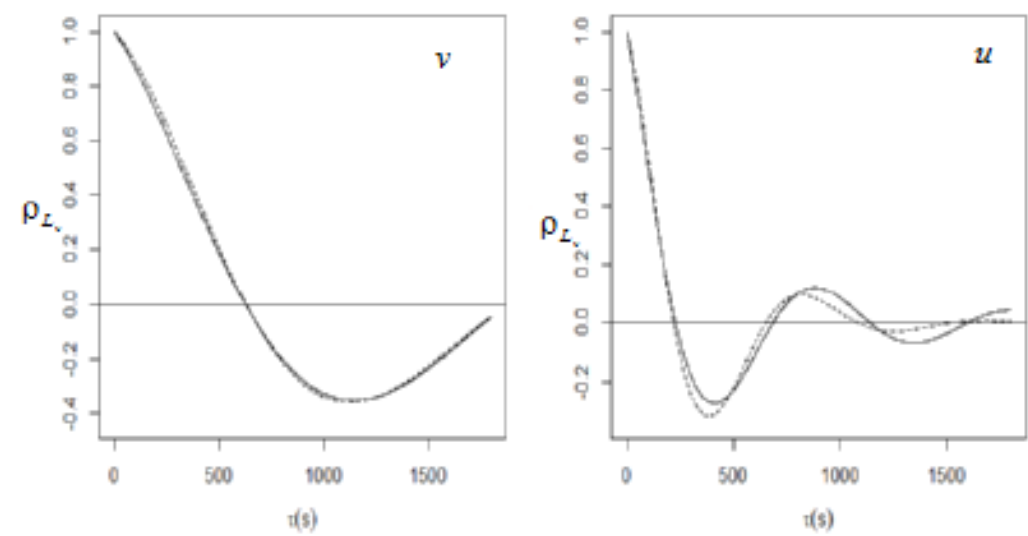

Figura 1 - Comparação entre as funções de autocorrelação para as componentes $u$ e $v$ o dia 21 de abril de 2001, 23 horas LT. A linha tracejada reproduz a função de autocorrelação de Frenkiel (Eq. (4)) e a linha contínua representa a função de autocorrelação de sugerida (Eq. (3).

\section{EQUAÇÃO DA VARIANÇA ESPACIAL LATERAL}

Partindo da Equação de Taylor para a variância espacial lateral,

$$
\sigma_{y}^{2}=2 \sigma_{v}^{2} \int_{0}^{t}(t-\tau) \rho_{L_{v}} d \tau
$$

substituindo a função de autocorrelação dada e pela Eq.(3), obtém-se

$$
\sigma_{y}^{2}=2 \sigma_{v}^{2} \int_{0}^{t}(t-\tau) \frac{\cos (q \tau)}{(1+p \tau)^{2}} d \tau
$$

Expandindo a função cosseno em série de MacLaurin e resolvendo as integrais resultantes, obtém-se

$$
\begin{aligned}
& \frac{\sigma_{y}^{2}}{2 \sigma_{v}^{2}}=\frac{-\cos (q t)}{p^{2}}+\frac{1+p t}{p^{2}}-\frac{q(1+p t)}{p^{3}} \times \\
& \times\left[\sum_{n=0}^{\infty} \quad\left(\frac{(p t)^{2 \mathrm{n}+2}}{2 \mathrm{n}+2}-\frac{(p t)^{2 \mathrm{n}+3}}{2 \mathrm{n}+3}\right)\left(\sum_{k=0}^{n} \quad(-1)^{n} \frac{m^{2 \mathrm{k}+1}}{(2 \mathrm{k}+1) !}\right)\right]- \\
& -\frac{1}{p^{2}}\left[\sum_{n=0}^{\infty} \quad\left(\frac{(p t)^{2 \mathrm{n}+1}}{2 \mathrm{n}+1}-\frac{(p t)^{2 \mathrm{n}+2}}{2 \mathrm{n}+2}\right)\left(\sum_{k=0}^{n} \quad(-1)^{n} \frac{m^{2 \mathrm{k}}}{(2 \mathrm{k}) !}\right)\right]
\end{aligned}
$$

\section{CONCLUSÃO}

A Eq. (6) obtida permitirá a obtenção de relações funcionais utilizadas em parametrizações turbulentas aplicadas em modelos de dispersão lagrangianos. Entretanto, a 
validade da Eq. (6) está condicionada ao cumprimento de critérios de validação da função de autocorrelação lagrangiana (Manomaiphiboon e Russel, 2003).

\section{REFERÊNCIAS}

TAYLOR, G. I. Diffusion by continuous movements, Proc. London Math. Soc. 20, 196-211, 1921.

HINZE, J. O. Turbulence, McGraw-Hill, New York, 1975.

PASQUILL, F., SMITH, F. B.. Atmospheric Diffusion. Ellis Horwood, 437, 1983.

KOLMOGOROV, A. N. The local structure of turbulence in incompressible viscous fluid for very large Reynolds numbers. In: Dokl. Akad. Nauk SSSR. 1941. p. 299-303.

MANOMAIPHIBOON, K.; RUSSELL, A. G. Evaluation of some proposed forms of Lagrangian velocity correlation coefficient. International journal of heat and fluid flow, v. 24, n. 5, p. 709-712, 2003.

PHILLIPS, P. and PANOFSKY, H.A. A re-examination of lateral dispersion from continuous sources, Atmospheric Environment. 16, 1851-1860, 1982.

FRENKIEL, F.N. Turbulent diffusion: mean concentration distribution in a flor field of homogeneous turbulence". Adv. Appl. Mech., 3, 1953, 61-107. 